\title{
Arbolado público en el barrio Centro, ciudad de Santiago del Estero. Estado actual y conformidad con la normativa municipal
}

\section{Downtown's Public trees in Santiago del Estero city. Actual state and compliance with legal normative}

\section{María Eugenia Arias ${ }^{1}$; Juan Pablo Celemín² y Andrea Alejandra Fuster ${ }^{3}$}

${ }^{1}$ Instituto de Geografía, Historia y Ciencias Sociales, Consejo Nacional de Investigaciones Científicas y Técnicas (CONICET), Universidad Nacional del Centro de la Provincia de Buenos Aires. Instituto del Hábitat y Ambiente, Facultad de Arquitectura, Urbanismo y Diseño, Universidad Nacional de Mar del Plata. Gral. Pinto 399, CP 7000, Tandil, Buenos Aires, Argentina.

${ }^{2}$ Instituto de Geografía, Historia y Ciencias Sociales, Consejo Nacional de Investigaciones Científicas y Técnicas (CONICET), Universidad Nacional del Centro de la Provincia de Buenos Aires.

${ }^{3}$ Instituto de Protección Vegetal, Facultad de Ciencias Forestales, Universidad Nacional de Santiago del Estero.

E-mail: me.arias@conicet.gov.ar

Arias, M. A., Celemín, J. P. \& Fuster, A. A. (2020). Arbolado público en el barrio Centro, ciudad de Santiago del Estero. Estado actual y conformidad con la normativa municipal. Revista Estudios Ambientales, 8(1), 95-114. 


\section{RESUMEN}

El arbolado es uno de los elementos más importantes en las ciudades, ya que los árboles forman parte integral del ambiente urbano y ofrecen numerosos beneficios ambientales, económicos, sociales y materiales. El objetivo de la investigación consistió, por un lado, en cotejar las plantaciones realizadas en el barrio Centro de la Ciudad de Santiago del Estero (Argentina) con la normativa vigente, y por el otro, establecer la cantidad de espacios verdes públicos y la superficie que ocupan. Para la primera parte del trabajo, se consideraron listas de especies botánicas sugeridas y no sugeridas por la ordenanza municipal. Se realizó un censo de vegetación considerando únicamente el arbolado de alineación del barrio Centro con un recorrido por sus 86 manzanas, durante los meses de verano del año 2019. Las especies vegetales censadas fueron identificadas hasta el nivel taxonómico de especie. Por su parte, las especies no sugeridas por la ordenanza fueron clasificadas según su origen biogeográfico y porte. El censo arrojó una abundancia de 3.125 ejemplares y una riqueza específica de 73 especies. De acuerdo al porte se reconocieron un mayor número de árboles, y por el origen se registraron mayor cantidad de especies exóticas. Además, se encontraron especies botánicas no recomendadas y recomendadas por la normativa. En relación a la segunda parte del trabajo, el barrio presentó un total de 11 espacios verdes públicos, los cuales representan el 1,87\% de la superficie total siendo considerada una superficie insuficiente. De la cantidad de espacios verdes un mayor porcentaje lo ocupan plazas y un menor porcentaje lo constituyen plazoletas.

Palabras clave: arbolado urbano, Santiago del Estero, ordenanza municipal, censo de vegetación, espacios verdes públicos.

\section{ABSTRACT}

Trees are one of the most important elements in cities, as trees are an integral part of the urban environment and offer numerous environmental, economic, social and material benefits. The objective of the research consisted, on one hand, in comparing the trees present in the Centro neighborhood of the City of Santiago del Estero (Argentina) with current regulations, and, on the other, establishing the amount of Public Green Spaces and the surface they occupy in the neighborhood. For the first 
part of the work, lists of botanical species suggested and not suggested by the municipal ordinance were considered. A vegetation census was carried out considering only the alignment trees of the Centro neighborhood consisting of 86 blocks, during the summer months of 2019. The trees were identified up to the taxonomic level of species. For their part, the species not suggested by the local ordinance were classified according to their biogeographical origin and size. The census showed an abundance of 3.125 specimens and a specific richness of 73 species. According to the size, a greater number of trees were recognized, and by origin, a greater number of exotic species were recorded. In addition, botanical species not recommended and recommended by regulations were found. In relation to the second part of the work, the neighborhood presented a total of 11 Public Green Spaces, which represent 1,87\% of the total area which is considered insufficient. Of the amount of green spaces, a greater percentage is occupied by squares and a smaller percentage is made up of small squares.

Keywords: urban trees, Santiago del Estero, municipal ordinance, vegetation census, public green spaces.

\section{INTRODUCCIÓN}

La vegetación urbana, especialmente los árboles, ofrece numerosos beneficios que pueden incrementar la calidad del ambiente y la salud humana tales como mejoras en la calidad del aire y del agua, la conservación de la energía, la regulación de la temperatura, las reducciones de la radiación ultravioleta, entre otros servicios ambientales (Conway y Bourne, 2013; Mills et al., 2015; Wang et al., 2016). El arbolado de alineación contiene a la población de árboles que están plantados en forma lineal en las veredas de calles y avenidas del área urbana y suburbana (Benedetti y Campo de Ferreras, 2007), y constituye el principal elemento vegetal que otorga biodiversidad a un medio construido. Por ello, el estudio de estas áreas verdes es clave para generar estrategias de gestión (Benedetti et al., 2016). Tal es su relevancia que la Organización Mundial de la Salud (OMS) estima que cada persona debe contar con un mínimo de 10 y hasta $15 \mathrm{~m}^{2}$ de espacio verde para vivir en un entorno saludable (Gómez Piovano y Mesa, 2015). 
Existen estudios sobre el arbolado urbano de la Ciudad de Santiago del Estero (e. $g$. Villaverde et al., 2010; Roger et al., 2016), donde se analiza la flora urbana cultivada evaluando la composición, abundancia, frecuencia relativa, usos asignados y conocimiento urbano asociado.

En la ciudad de Santiago del Estero, la normativa referente al Arbolado Urbano se encuentra vigente en la Ordenanza $N^{\circ} 3.823 / 2004$. En base a este cuerpo legal, se expresa una definición de arbolado urbano y los objetivos de dicha ordenanza:

Se define como Arbolado Urbano a toda población arbórea, arbustiva y herbácea; instalada en los espacios del dominio Público y Privado Municipal, insertos en el ámbito de su ejido. Los objetivos de esta ordenanza son la conservación y el acrecentamiento de la población vegetal existente por implantarse en el futuro, de acuerdo a lo expresado en los artículos $41^{\circ}-42^{\circ}$ $43^{\circ}$, referidos a la Conservación y Defensa del Medio Ambiente surgidos de la Constitución Nacional Argentina.

La Ordenanza №3.823/2004 en su Título I Disposiciones Generales, establece la obligatoriedad del arbolado en veredas del ejido municipal de 1,50 m o más de ancho, y arbolado optativo en caso de un ancho de vereda inferior.

Asimismo, la Ordenanza municipal en su Título II Disposiciones especiales o Reglamentarias - De las Plantaciones, apartado a), expresa que es obligación de los frentistas, colocar por lo menos un árbol cada 5 metros de vereda, dejando libres las ochavas.

Por otro lado, dicha ordenanza en su apartado c), establece que:

Se prohíbe en las veredas, la plantación de especies arbóreas que por su porte y morfología en general; resultarán dañosas en el futuro o potencialmente peligrosas: todas las especies del género Populus, Eucaliptos, todas las especies del género Ficus, Palo borracho, Pacará, Mora negra o blanca, Mora turca, Casuarinas, Olmos, Sauces, Palmeras (todas las variedades), etc. Sobre especies a plantar, técnicas de plantación, épocas de plantación, etc., se seguirán las sugerencias del Área de Producción Forestal y Arbolado Urbano.

Cabe mencionar la Ordenanza $N^{\circ} 3.385 / 2000$ sobre Nómina de Espacios Verdes Consolidados de la Ciudad de Santiago del Estero. Esta normativa referida a los espacios verdes públicos (EVP), los clasifica en cuatro categorías: Parques, Plazas, Plazoletas o Isletas y Paseos o Bulevares. 
El trabajo se divide en dos partes principales. La primera corresponde al análisis de las especies arbóreas del barrio Centro para determinar si se adecuan a la normativa municipal vigente. La segunda corresponde a un relevamiento de los espacios verdes del área de estudio para establecer su distribución y características.

\section{Área de estudio}

El área de estudio corresponde al barrio Centro, que se encuentra ubicado en la ciudad de Santiago del Estero, capital de la provincia homónima y cabecera del Departamento Capital (Fig. 1).

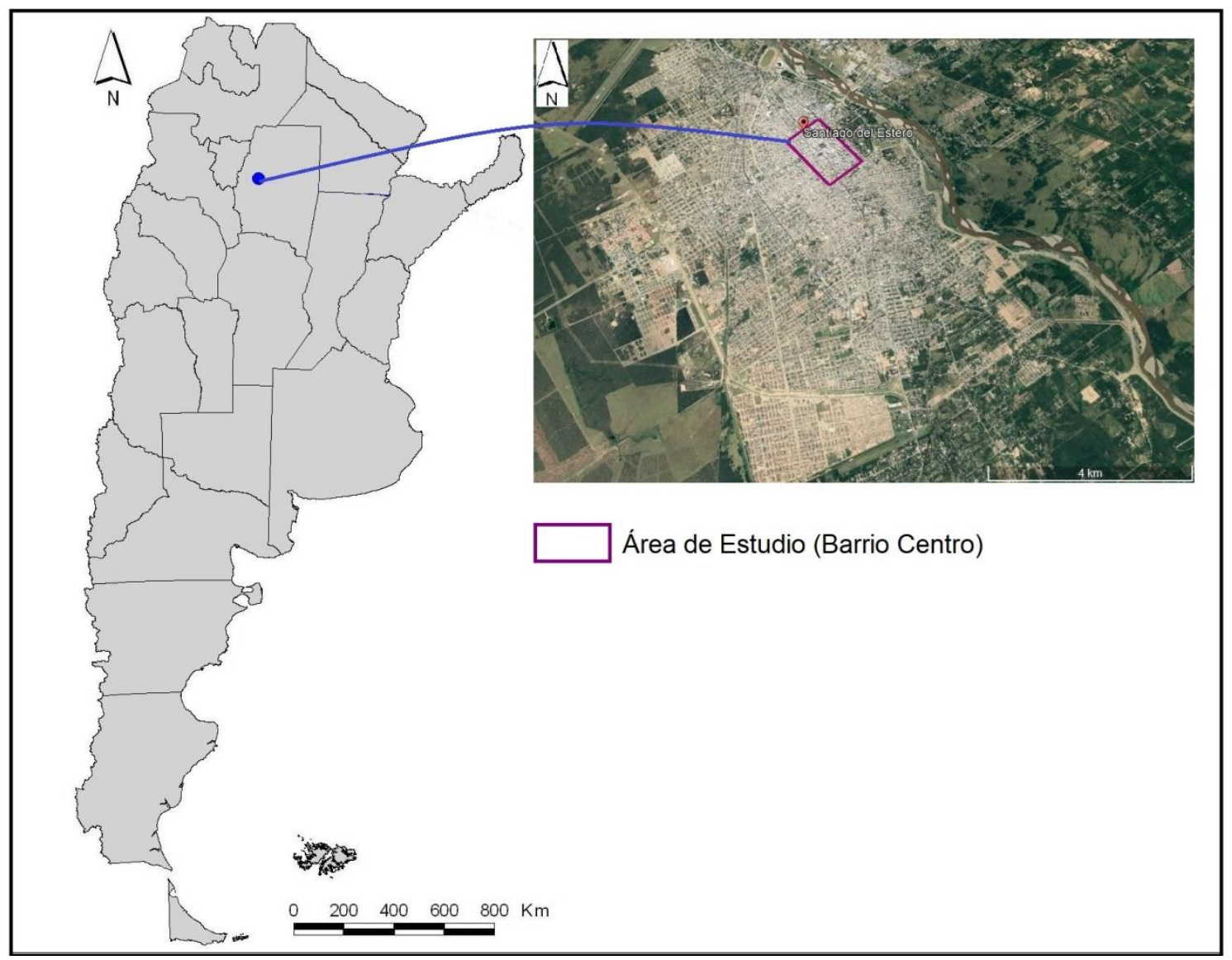

Figura 1: Ubicación del área de estudio en la República Argentina, Santiago del Estero, Dpto. Capital. Fuente: elaboración personal en base a cartografía propia e imágenes de Google Earth Pro obtenida el 20/04/20.

La ciudad de Santiago del Estero está situada entre $27^{\circ} 47^{\prime} 04^{\prime \prime}$ S y $64^{\circ} 16^{\prime} 01^{\prime \prime} \mathrm{O}$, sobre la margen derecha del río Dulce, a una altitud de 182 m s.n.m. 
El departamento Capital tiene una superficie de $2.116 \mathrm{~km}^{2}$ (1,5\% del total provincial) y según el último censo nacional realizado en 2010 viven 267.125 personas (30,56\% del total provincial) de las cuales 232.796 se encuentran en zona urbana. La densidad poblacional es de 126,24 hab/km² (INDEC, 2012). En este departamento la mayor parte de la población se concentra en esta ciudad, que es el centro administrativo, financiero y comercial de la provincia.

Por su parte el barrio Centro (Fig.2) de la ciudad de Santiago del Estero está localizado entre las avenidas: Rivadavia, Moreno, Alsina y Roca. Alcanza un perímetro de $5,10 \mathrm{~km}$ y un área de $1,55 \mathrm{~km}^{2}$.

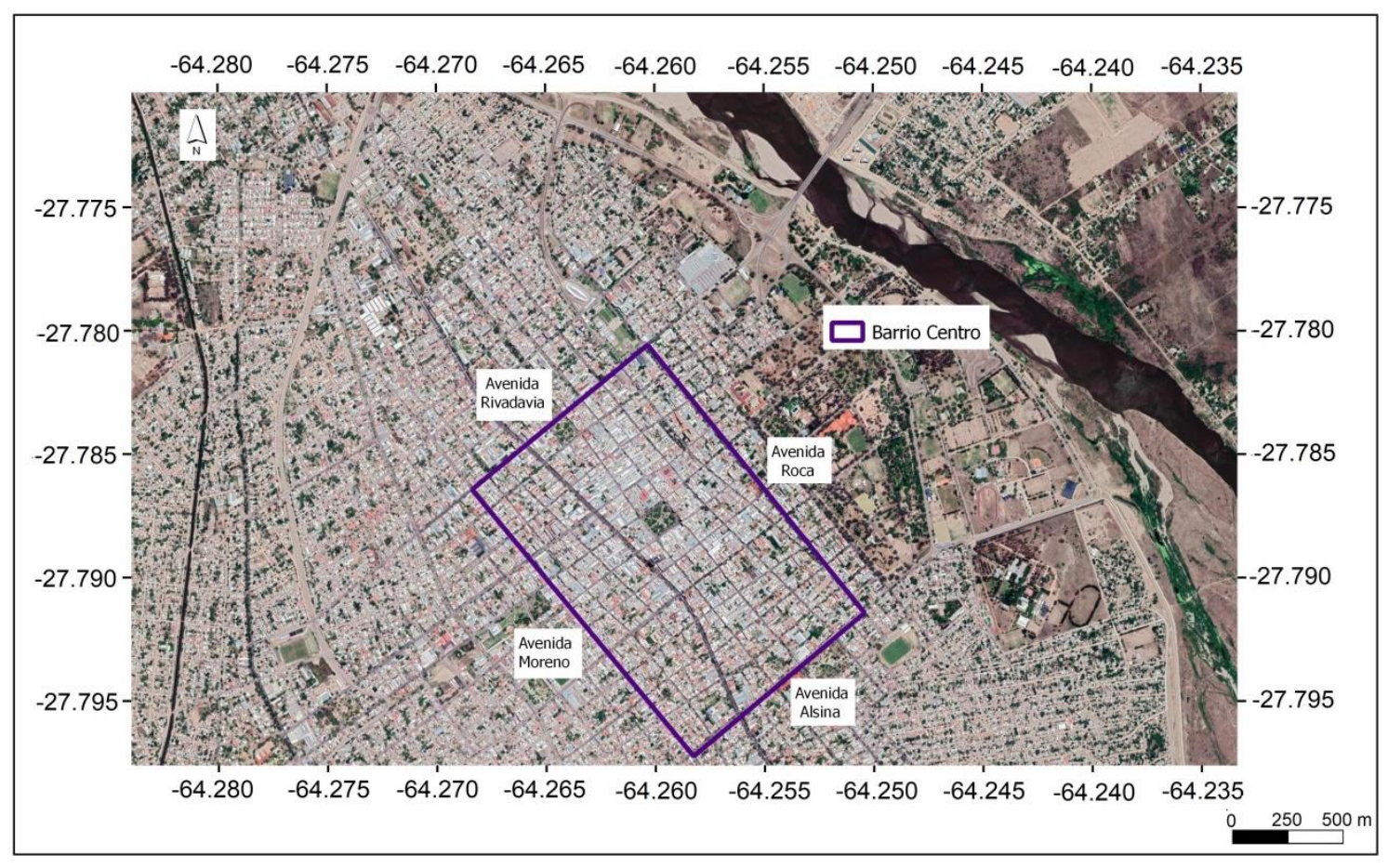

Figura 2: Ubicación del barrio Centro en la ciudad de Santiago del Estero. Fuente: elaboración personal en base a imágenes de Google Earth Pro obtenida el 20/04/20.

El barrio Centro se seleccionó como área de estudio en primer lugar por ser las zonas céntricas de las ciudades las que menos espacios verdes y árboles ofrecen a sus habitantes debido a la alta densidad edilicia. Otro motivo de elección, es que se trata de una zona donde hay una mayor concentración de tránsito vehicular, siendo esta la principal fuente de contaminación atmosférica (emanación de gases) y sonora. Por último, se consideró este sitio debido al elevado consumo de energía derivado de las múltiples actividades que se realizan en ese sector. 


\section{METODOLOGIA}

Se llevó a cabo un censo de especies vegetales en el barrio Centro de la ciudad de Santiago del Estero en los meses de verano del año 2019, recorriendo a pie 86 manzanas, cada una de las cuales correspondió a una unidad muestral. El censo consideró únicamente a los árboles que se disponen en las aceras y no fueron contemplados los que se hallan en los espacios verdes. En un plano del barrio se colocó un número de identificación para cada manzana y se indicaron las secciones (calles) que conforman la manzana mediante las letras a, b, c y d, siguiendo el sentido de las agujas del reloj. Las especies situadas en las esquinas de cada manzana fueron tomadas como punto de inicio del censo (Fig. 3).

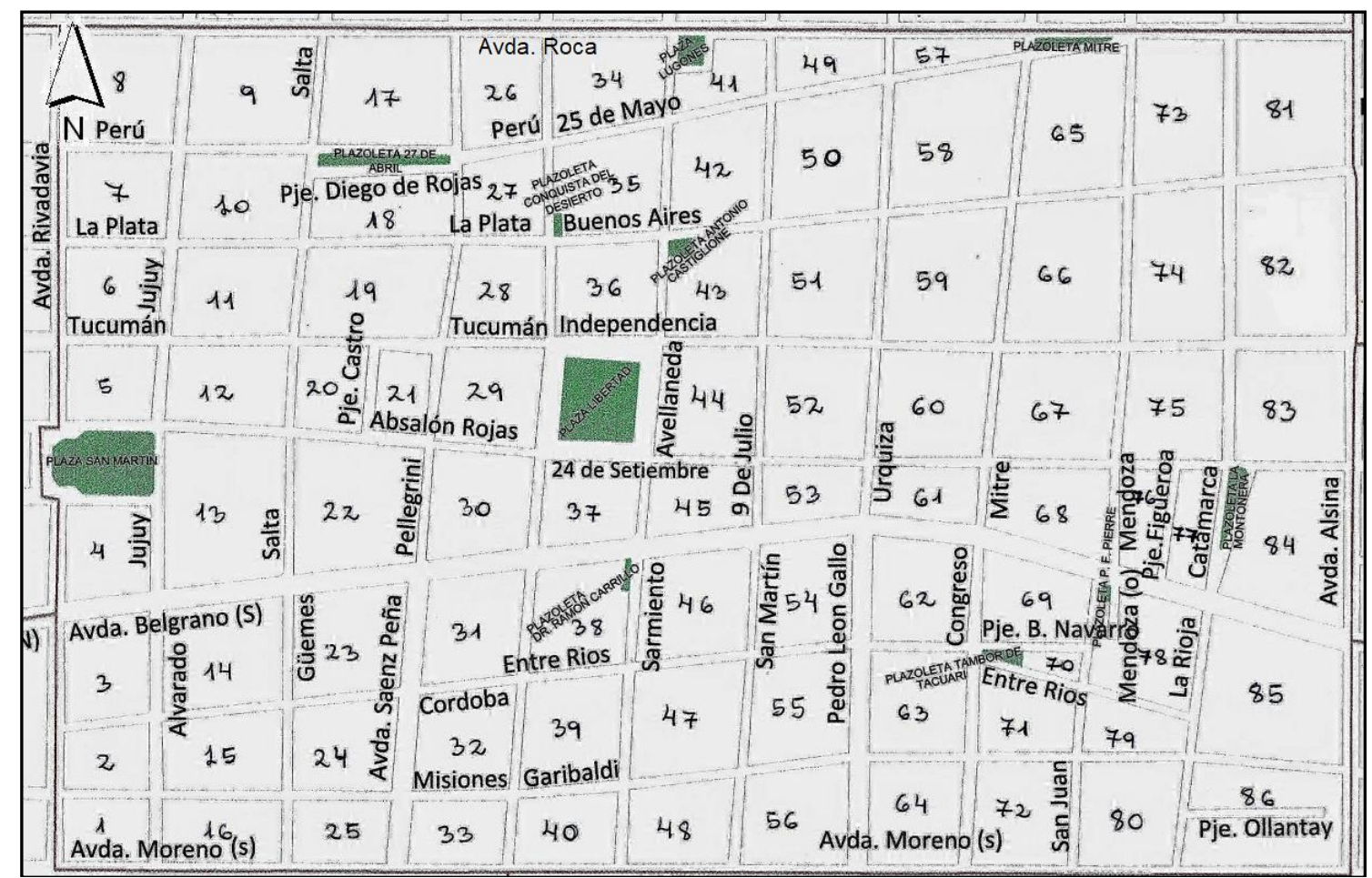

Figura 3: Plano modificado para realizar el censo en el barrio Centro, Santiago del Estero. Fuente: Elaboración propia en base al plano de la ciudad provisto por la Subsecretaría de Planeamiento, Municipalidad de la Capital.

Se determinó la abundancia absoluta mediante conteo directo de individuos y se estimó la riqueza específica, que se basa en el número de especies presentes, sin tomar en cuenta el valor de importancia de las mismas (Magurran, 1988; Moreno, 2001). 
Las especies botánicas censadas fueron identificadas a nivel taxonómico de especie mediante claves botánicas y bibliografía especializada (e. g. Roic y Villaverde, 1999; Villaverde y Villaverde, 2005; Villaverde et al., 2010; Grau y Kortsarz, 2012; Roger et al., 2014; Palacio y Roger, 2016). Según el origen biogeográfico los individuos fueron agrupados en nativos (cuando su área de distribución incluye a la provincia de Santiago del Estero), no nativos (cuando son originarios de otras regiones fitogeográficas del país) y exóticos (originarios de otros países) (Roger et al., 2016). En cuanto al porte se clasificó en árboles, arbustos, palmeras y plantas herbáceas o hierbas. Demaio et al. (2002:20) definen al árbol como "vegetales leñosos que desarrollan un tronco principal perenne de al menos $7 \mathrm{~cm}$ de diámetro a la altura de pecho $(1,3 \mathrm{~m})$ y una altura total de por lo menos $4 \mathrm{~m}$ " y se define como arbusto a las plantas leñosas, no superiores a $5 \mathrm{~m}$ de altura y ramificadas desde su base (Roger et al., 2016). Las palmeras son plantas que sólo tienen crecimiento primario, es decir son plantas monocotiledóneas con porte de estípite con un sistema radicular fasciculado (Ferro, 2004). En tanto que las hierbas son plantas no lignificadas de consistencia blanda en todos sus órganos (Giménez y Moglia, 2003).

\section{RESULTADOS Y DISCUSIÓN}

Especies botánicas identificadas en el barrio Centro

El arbolado de alineación del barrio Centro tiene una abundancia de 3.125 ejemplares con una riqueza específica de 73. En el estudio realizado por Villaverde et al. (2010), se observó un mayor valor de abundancia $(\mathrm{N}=3.862)$ y un menor valor de riqueza específica $(S=61)$ datos que fueron obtenidos del censo llevado a cabo en el barrio durante el año 2007. Esto pone en evidencia como cambió la composición florística en el área de estudio. Las especies identificadas pertenecen a 62 géneros, 33 familias y 23 órdenes. Del total de especies, 48 son arbóreas (66\%), 19 son arbustivas (26\%), 5 son palmeras (7\%) y sólo una es herbácea (1\%) (Fig. 4).

En lo referido al origen biogeográfico, se registraron 50 especies exóticas con una representatividad del $68,50 \%$; le sigue una menor cantidad de especies no nativas (19), con una representatividad del $26,02 \%$ y por último solo 4 especies nativas, las que se encuentran escasamente representadas (5,48\%) (Fig. 5). 
En relación al número de individuos según el tipo de porte, se hallaron 2.640 árboles, seguido de 385 arbustos, 99 palmeras y 1 planta herbácea (Fig. 6).

La abundancia según el origen biogeográfico muestra una mayor cantidad de especies exóticas ( $\mathrm{N}=1.965$ ), seguido por 1.154 especies no nativas y 6 nativas (Fig. 7).

Las especies exóticas más abundantes son: Citrus x aurantium L., Ligustrum lucidum W.T. Aiton., Thevetia peruviana (Pers.) K. Schum, Brachychitonpopulneus (Schott y Endl.) R.Br., Fraxinus pennsylvanica Marshall. yLagerstroemia indica (L) Pers. Las especies de plantas no nativas más abundantes son: Handroanthus impetiginosus (Mart. ex DC.) Mattos, Jacaranda mimosifolia D. Don., Tipuana tipu (Benth.) Kuntze, Sapindus saponaria L. y Syagrus romanzoffiana (Cham.) Glassman. Las especies autóctonas presentes son: Geoffroea decorticans (Gill. ex Hook. et Arn.) Burkart, Acacia aromaGillies ex Hook.etArn., Ceiba speciosa (A.St.-Hil.) Ravenna y Jodina rhombifolia (Hook. etArn.) Reissek.En el Gran San Miguel de Tucumán se elaboró un listado de especies vegetales que han sido probadas como las más adecuadas para arbolado urbano. Las especies Lagerstroemia indica, Thevetia peruviana, Citrus x aurantium pertenecen al grupo de especies con dimensiones reducidas que son útiles cuando hay restricciones de espacio y cableado. En el otro grupo de especies intermedias que tienen tamaño mediano a grande y son aptas para veredas y calles con mayor espacio y avenidas se encuentran Fraxinus pennsy/vanica, Jacaranda mimosifolia, Handroanthus impetiginosusy Brachychiton populneus. En el último grupo se incluye a las especies que deberían restringirse a avenidas y espacios públicos amplios, tal es el caso de Tipuana tipu. Como especies adecuadas para regiones semiáridas de la provincia de Tucumán, se hallan Lagerstroemia indica y Tipuana tipu (Grau y Kortsarz, 2012). Cabe mencionar que varias de las especies censadas en el barrio Centro, coinciden con las especies vegetales de este listado.

El Ministerio de Medio Ambiente de la Provincia de Santa Fe (2017) realizó un listado de especies nativas usadas en el arbolado para veredas angostas en la provincia de Santa Fe y determinó que las especies Geoffroea decorticans, Acacia aroma, Ceiba speciosa y Jodina rhombifoliano son habitualmente empleadas en arbolado de calle y, en general no se reproducen en los viveros comerciales debido a las características morfológicas que poseen tales como espinas, aguijones y hojas espinosas. Probablemente estas son las causas porque esas especies no son cultivadas en el barrio Centro de Santiago del Estero. 


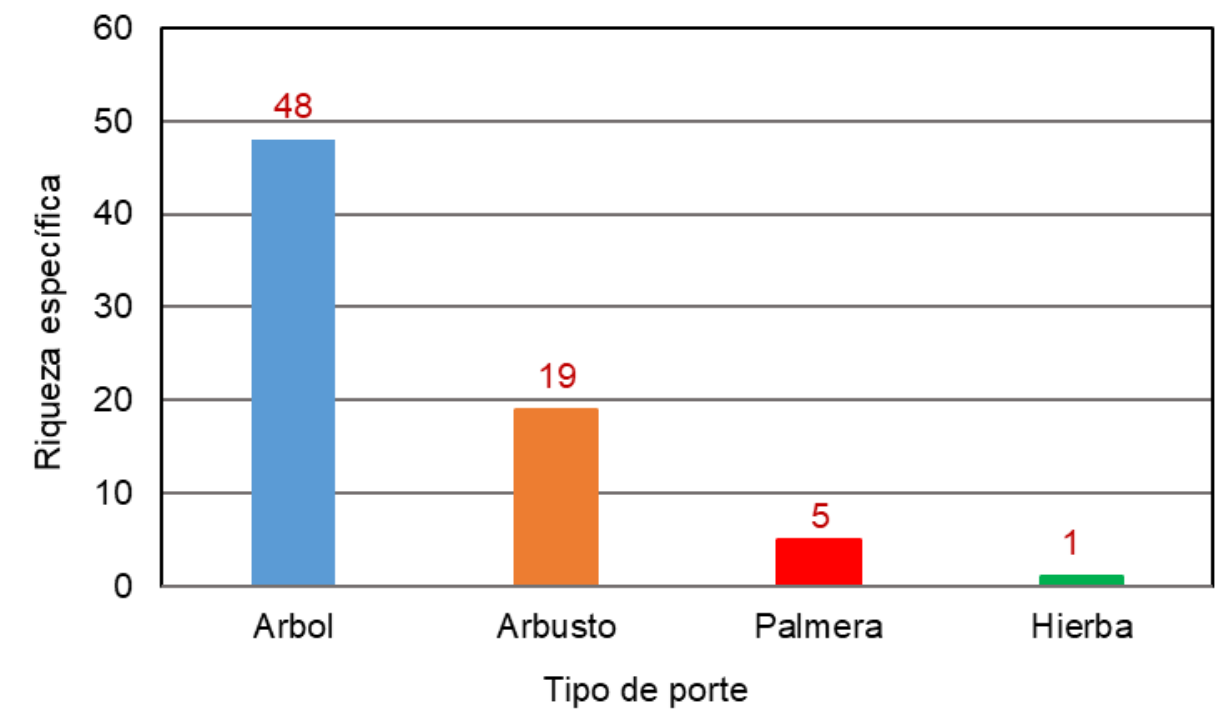

Figura 4: Riqueza de especies botánicas según el porte en el barrio Centro de la ciudad de Santiago del Estero. Fuente: elaboración propia.

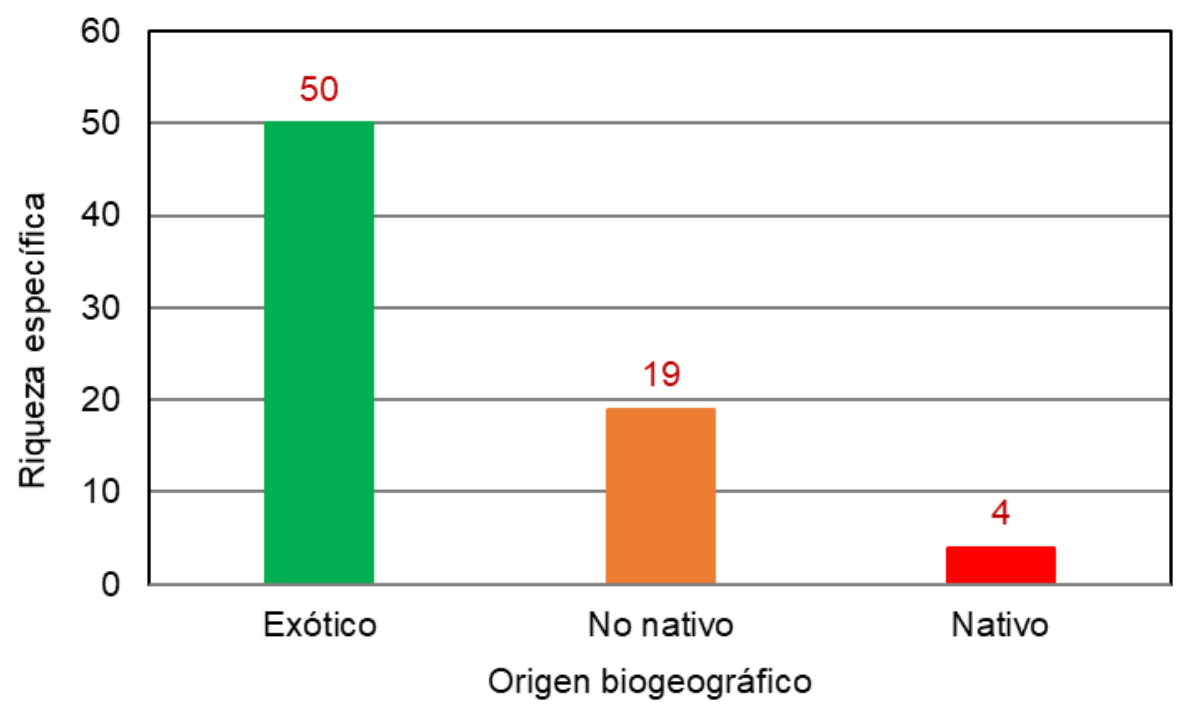

Figura 5: Riqueza de especies botánicas según el origen biogeográfico en el barrio Centro de la ciudad de Santiago del Estero. Fuente: elaboración propia. 


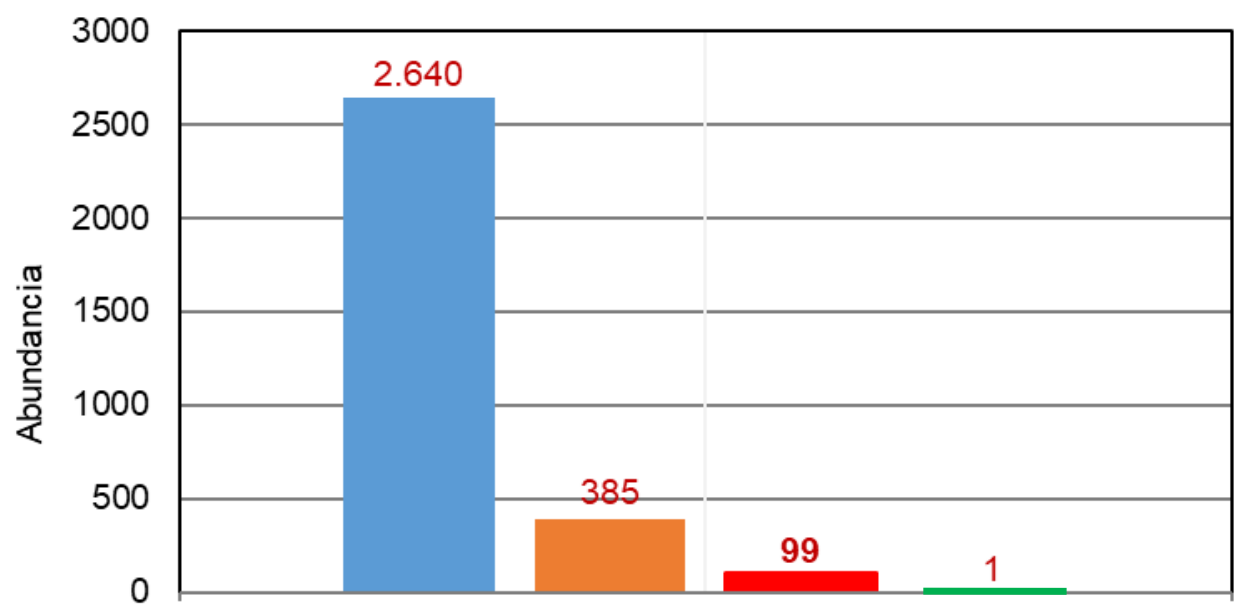

Tipo de porte

arbol $n$ Arbusto $\square$ Palmera $\square$ Hierba

Figura 6: Cantidad de árboles, arbustos, palmeras y herbáceas presentes en el arbolado del barrio Centro de la ciudad de Santiago del Estero. Fuente: elaboración propia.

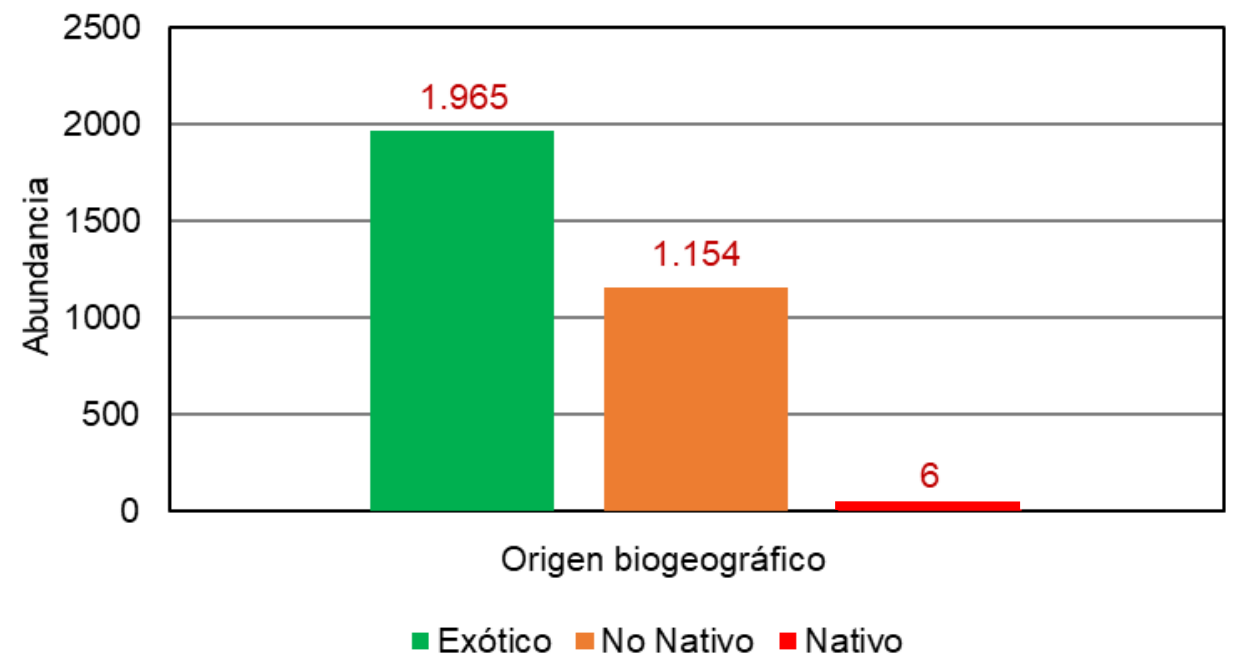

Figura 7: Cantidad de exóticas, nativas y no nativas presentes en el arbolado del barrio Centro de la ciudad de Santiago del Estero. Fuente: elaboración propia.

Adecuación a la Ordenanza N³.823/2004

Según la normativa debieran existir en el arbolado del barrio Centro 5.472 ejemplares, estimando 16 árboles por cuadra (100 o 150 metros). Sin embargo, de acuerdo al censo realizado la abundancia obtenida es de 3.125 ejemplares. Por lo tanto, el número de individuos existentes en las veredas no se adecua a lo que establece la 
ordenanza municipal, faltando unos 2.347 ejemplares para ser plantados en los espacios libres disponibles en las aceras.

La Municipalidad de Santiago del Estero, a partir de la citada ordenanza recomienda una lista de especies botánicas a utilizar en el arbolado de alineación según el ancho de las veredas (Tabla 1), de las cuales sólo se identificaron 19 especies en el presente estudio.

Tabla 1. Especies botánicas sugeridas por la Municipalidad de Santiago del Estero

\begin{tabular}{|c|c|c|}
\hline \multicolumn{3}{|c|}{ Nombre de especies botánicas sugeridas según ancho de vereda } \\
\hline Veredas de 1,5 a $2,5 \mathrm{~m}$ & Veredas de 2,5 a $3 \mathrm{~m}$ & Veredas de más de $3 \mathrm{~m}$ \\
\hline $\begin{array}{l}\text { Citrus x aurantium } \mathrm{L} . \\
\text { "naranjo agrio" }\end{array}$ & $\begin{array}{l}\text { Geoffroea decorticans } \\
\text { (Gill. ex Hook. et Arn.) } \\
\text { Burkart "chañar" }\end{array}$ & $\begin{array}{c}\text { Handroanthus } \\
\text { impetiginosus (Mart. ex } \\
\text { DC.) Mattos. "lapacho } \\
\text { rosado" }\end{array}$ \\
\hline $\begin{array}{l}\text { Lagerstroemia indica L. } \\
\text { "crespón" }\end{array}$ & $\begin{array}{c}\text { Handroanthus } \\
\text { impetiginosus (Mart. ex } \\
\text { DC.) Mattos. "lapacho } \\
\text { rosado" }\end{array}$ & $\begin{array}{l}\text { Melia azedarach L. } \\
\text { "paraíso sombrilla" }\end{array}$ \\
\hline $\begin{array}{l}\text { Ligustrum lucidum W.T. } \\
\text { Aiton "ligustro" }\end{array}$ & $\begin{array}{l}\text { Senna spectabilis (DC.) } \\
\text { H.S. Irwin \& Barneby. } \\
\text { "carnaval" }\end{array}$ & $\begin{array}{l}\text { Grevillea robusta A. Cunn. } \\
\text { ex R. Br. "grevilea" }\end{array}$ \\
\hline $\begin{array}{c}\text { Prunus domestica L. } \\
\text { "ciruelo" }\end{array}$ & $\begin{array}{l}\text { Fraxinus pennsylvanica } \\
\text { Marshall. "fresno rojo } \\
\text { americano" }\end{array}$ & $\begin{array}{l}\text { Acacia aroma Gillies ex } \\
\text { Hook. et Arn. "tusca" }\end{array}$ \\
\hline $\begin{array}{l}\text { Bauhinia variegata L. } \\
\text { "pata de vaca" }\end{array}$ & $\begin{array}{c}\text { Sapindus saponaria L. } \\
\text { "palo jabón" }\end{array}$ & $\begin{array}{c}\text { Jacaranda mimosifolia D. } \\
\text { Don. "jacarandá" }\end{array}$ \\
\hline Acacia sp. & $\begin{array}{c}\text { Brachychiton populneus } \\
\text { (Schott \&Endl.) R.Br. } \\
\text { "árbol botella" }\end{array}$ & $\begin{array}{l}\text { Tipuana tipu (Benth.) } \\
\text { Kuntze. "tipa blanca" }\end{array}$ \\
\hline $\begin{array}{c}\text { Thevetia peruviana } \\
\text { (Pers.) K. Schum "tevetia } \\
\text { (flor amarilla)" }\end{array}$ & & $\begin{array}{c}\text { Pterogyne nitens Tul. "tipa } \\
\text { colorada" }\end{array}$ \\
\hline $\begin{array}{l}\text { Thevetia peruvianaf. } \\
\text { aurantiaca H. St. John. } \\
\text { "tevetia (flor morada)" }\end{array}$ & & $\begin{array}{l}\text { Schinus areira L. } \\
\text { "aguaribay" }\end{array}$ \\
\hline
\end{tabular}

Fuente: elaboración propia en base a Ordenanza Municipal № 3.823 /2004.

En el área de estudio se registraron 17 especies no recomendadas por la ordenanza de las cuales 10 son árboles exóticos, un árbol es nativo y cinco son palmeras. De éstas últimas una especie es autóctona de Argentina y el resto son exóticas. 
En base a lo que establece la ordenanza municipal en su Título II apartado c), se presenta a continuación, la nómina de las especies no utilizables con su respectiva abundancia encontradas en el arbolado de alineación del barrio Centro (Tabla2).

Tabla 2. Especies botánicas no sugeridas por la ordenanza municipal identificadas en el área de estudio. Fuente: elaboración propia en base a Ordenanza Municipal № 3.823 /2004

\begin{tabular}{|c|c|}
\hline Nombre de especies no sugeridas & $\begin{array}{c}\text { Número de } \\
\text { individuos } \\
\text { encontrados en el } \\
\text { barrio Centro }\end{array}$ \\
\hline $\begin{array}{c}\text { Populus deltoides W. Bartram ex Marshall. "álamo } \\
\text { carolina" }\end{array}$ & 8 \\
\hline Ficus benjamina L. "boj o laurel de la India" & 37 \\
\hline Ficus lyrata Warb. "gomero pandurata" & 4 \\
\hline $\begin{array}{c}\text { Ceiba speciosa (A.St.-Hil.) Ravenna "palo borracho o } \\
\text { palo rosado" }\end{array}$ & 2 \\
\hline Morus alba L. "mora blanca" & 4 \\
\hline Morus xhybrida "mora híbrida" & 6 \\
\hline Broussonetia papyrifera (L.) L'Herit. "mora turca" & 15 \\
\hline Casuarina cunninghamianaMiq. "casuarina" & 13 \\
\hline Ulmus pumila L. "olmo siberiano" & 10 \\
\hline Salix babylonica L. "sauce llorón" & 3 \\
\hline Grevillea robusta A. Cunn. ex R. Br."grevilea" & 35 \\
\hline $\begin{array}{c}\text { Syagrus romanzoffiana (Cham.) Glassman "palmera } \\
\text { pindó" }\end{array}$ & 68 \\
\hline $\begin{array}{c}\text { Phoenix canariensis hort. ex Chabaud. "fénix o palma } \\
\text { de las Canarias" }\end{array}$ & 26 \\
\hline Roystonea regia (Kunth) O.F.Cook. "palmera real" & 1 \\
\hline Dypsis lutescens (H. Wendl.) Beentje \& J. Dransf. \\
"areca o palma de frutos de oro" & 3 \\
\hline $\begin{array}{c}\text { Phoenix roebelenii O'Brien "palmera enana o palmera } \\
\text { de Roebelen" }\end{array}$ & 1 \\
\hline
\end{tabular}

Las especies vegetales no recomendadas por la normativa para ser utilizadas en arbolado de alineación poseen ciertas características estructurales que la convierten en no aptas para el arbolado urbano. Los árboles de gran altura y copa amplia pueden llegar a interferir con el cableado lo que hace necesario su poda. Otro aspecto que tienen algunas especies arbóreas es el gran desarrollo del sistema radicular, lo que puede generar daños en veredas y obstruir cañerías. En ciertos árboles se produce la caída de muchos frutos durante el período de maduración, que se acumulan y 
descomponen en las veredas (e. g. Morus alba y Morus nigra). También se encuentran árboles que presentan una copa irregular y abierta, con ramas quebradizas que al caer pueden generar daños materiales (Grau y Kortsarz, 2012). Asimismo, los árboles caducifolios, cuya pérdida de follaje se realiza de manera masiva, ocasionan una acumulación de hojas sobre el suelo lo cual genera molestia a los vecinos. Los árboles que se caracterizan por tener una copa extendida y ramas principales largas y arqueadas que llegan a tocar el suelo, como es el caso del Salix babylonica (Méndez, 2012), obstaculizan el paso de los transeúntes. Cabe mencionar que ciertas especies arbóreas tienen una madera muy blanda quedando expuesta al ataque de insectos taladradores y organismos fúngicos, lo cual puede provocar la caída de ramas y del propio ejemplar. Además, se puede señalar la característica de la especie nativa Ceiba speciosa, que, debido a poseer un tronco voluminoso cubierto por aguijones, no es recomendable su colocación en veredas. En el caso de las palmeras, una característica negativa para su cultivo en ciudades es que produce grandes cantidades de frutos lo cual puede generar un problema de limpieza (Grau y Kortsarz, 2012). Esto se evidencia con las especies Syagrus romanzoffiana y Phoenix canariensis. Igualmente debido a que las palmeras proporcionan una escasa sombra, no se aconseja su plantación en ciudades de clima árido.

\section{Espacios verdes públicos en el barrio Centro de la ciudad de Santiago del Estero}

El barrio Centro presenta un total de 11 espacios verdes públicos (Fig. 8), entre los cuales se identificaron las categorías plaza y plazoleta según la Ordenanza $\mathrm{N}^{\circ}$ 3.385/2000. Existen dos plazas que abarcan una superficie de $20.505 \mathrm{~m}^{2}$ un $69 \%$ de la superficie total de espacios verdes. Las plazoletas ocupan $9.119 \mathrm{~m}^{2}$ conformando un $31 \%$ de la superficie total. Las plazas Libertad y San Martín son las que tienen una mayor superficie (Fig. 9) con un $41 \%$ y $28 \%$ respectivamente. La plazoleta 27 de abril le sigue en orden de importancia con un $11 \%$ sobre la superficie total de espacios verdes. El resto de los espacios con menor superficie (Fig. 9) corresponden a 8 plazoletas: Cnel. Lorenzo Lugones con un 5\%; Bartolomé Mitre y La Montonera con un 4 \%; Dr. Ramón Carrillo, Dr. Antonio Castiglione y Tambor de Tacuarí con un 2\%; por último, Pbro. Pedro Fills Pierre y Conquista del Desierto con el $1 \%$.

Cabe mencionar que, en la ciudad de Santiago del Estero, hay escasa información disponible respecto a la composición florística vegetativa de los espacios verdes (Roic 
y Villaverde, 1999). Solamente se hicieron relevamientos de flora en las plazas Libertad y San Martín.

Teniendo en cuenta que la superficie del barrio Centro es de $1.578 .636 \mathrm{~m}^{2}$, la superficie ocupada por espacios verdes en el mismo es igual a $29.624 \mathrm{~m}^{2}$. Esto significa que los espacios verdes públicos representan únicamente el 1,87\% de la superficie total del área de estudio.

Respecto a la composición florística leñosa de la plaza Libertad el estudio de Roger et al. (2014) revela una importante diversidad vegetal que incluye especies arbóreas y arbustivas, y según su origen biogeográfico se encuentran tanto especies nativas como no nativas y exóticas. Además, destaca la predominancia de las exóticas sobre las especies no nativas y nativas.

Acerca de la composición florística de la plaza San Martín, las especies vegetales predominantes son las arbustivas seguidas de las arbóreas, siendo dominantes Ligustrum lucidum y Handroanthus impetiginosus respectivamente (Roger et al., 2012. Inédito).

Cabe señalar que de todas las plazoletas aquellas que se caracterizan por presentar en su predio escasa presencia de vegetación son: Dr. Antonio Castiglione, Conquista del Desierto y Dr. Ramón Carrillo. Mientras el resto evidencia una importante cantidad de ejemplares arbóreos y arbustivos. 


\section{revista estudios ambientales}

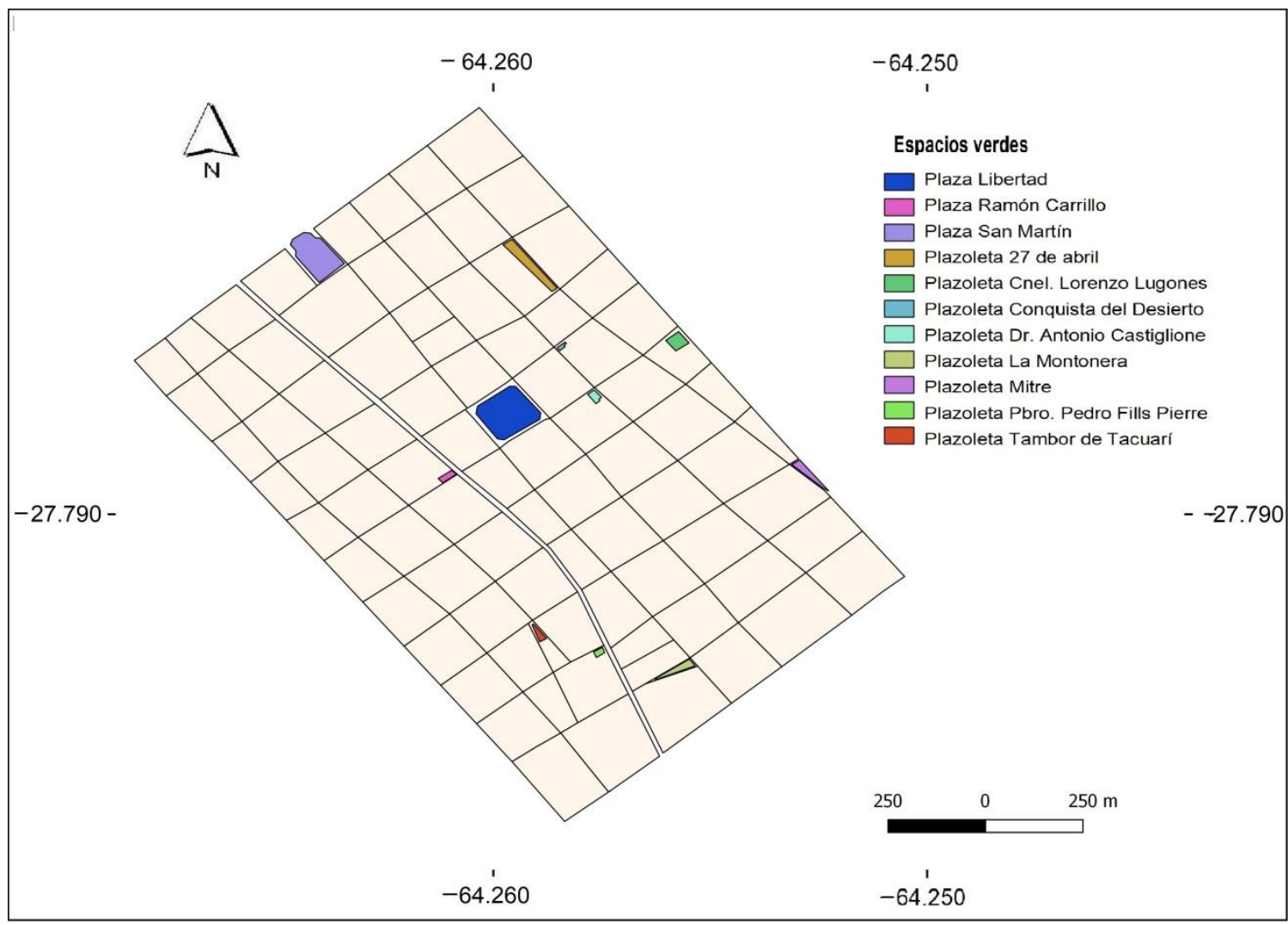

Figura 8: Espacios verdes públicos en el barrio Centro de Santiago del Estero. Fuente: elaboración propia.

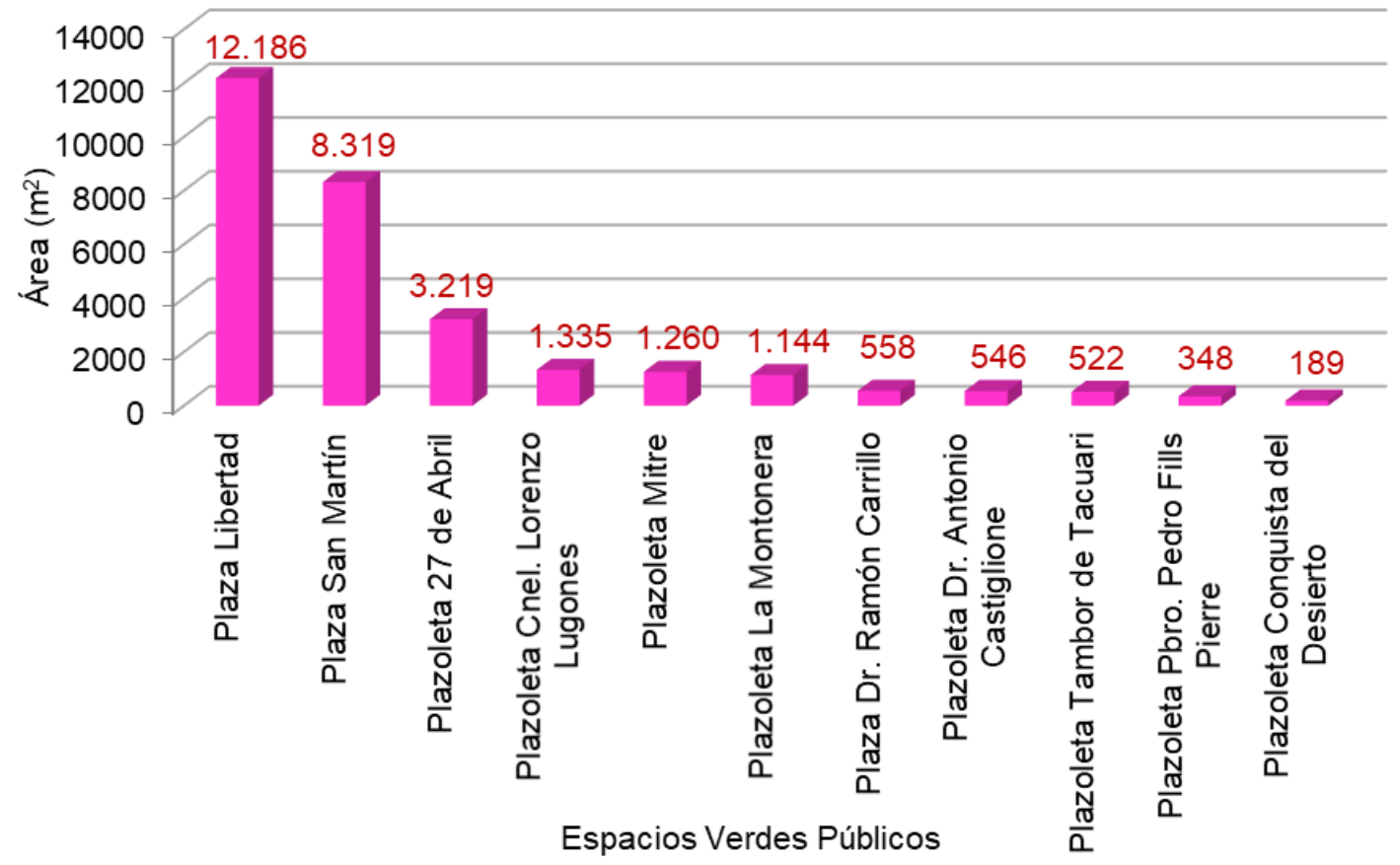

Figura 9: Superficie de los espacios verdes públicos en el barrio Centro de Santiago del Estero. Fuente: elaboración propia. 


\section{CONCLUSIONES}

En relación a la composición florística del barrio Centro la misma posee una gran cantidad de ejemplares y una alta riqueza de especies.

De acuerdo a los resultados obtenidos sobre el origen biogeográfico de las especies botánicas, se evidenció un elevado número de especies exóticas, seguido por las especies no nativas y un ínfimo valor de especies nativas. A su vez, la abundancia de las especies exóticas fue mayor que la correspondiente a las especies no nativas y nativas. Esto pone de manifiesto la preferencia por las especies botánicas exóticas, o bien especies de plantas originarias de otros lugares del país, dejando de lado la elección de especies locales para la plantación en el arbolado de alineación.

Por otro lado, según los resultados expuestos acerca del porte de las especies botánicas relevadas se encontró un mayor número de especies arbóreas, seguido por pocas especies arbustivas, mínima variedad de palmeras y solamente una especie herbácea. En cuanto a la abundancia presentada en cada tipo, se observó una mayor cantidad de árboles en relación a los tipos restantes. Estos resultados son positivos ya que los árboles al tener una morfología y capacidad adecuada para brindar un mayor número de beneficios ambientales es aconsejable su uso en veredas.

El arbolado urbano público del barrio Centro no se ajusta a la Ordenanza $\mathrm{N}^{\circ}$ $3.823 / 2004$ en lo que respecta a plantaciones en las veredas. La cantidad de individuos en el arbolado de alineación no es el suficiente, ya que se estimó un faltante del 42,89 \% proporción considerable que deber ser completada con la plantación de más ejemplares. Igualmente, del inventario se obtuvieron especies botánicas no sugeridas para su uso en el arbolado de alineación.

Respecto a los espacios verdes públicos existentes en el barrio, éstos son insuficientes y sus respectivas superficies son reducidas en relación a la superficie total del mismo. Asimismo, se evidencia una inadecuada distribución de estos espacios en el interior del área de estudio.

Incorporarlos beneficios del arbolado de alineación en los planes de desarrollo urbanos dará lugar al establecimiento de políticas de gestión sustentable en las que se asigne un valor ambiental a los árboles. De esta manera se podrá alcanzar la sustentabilidad en el manejo del arbolado urbano. 


\section{BIBLIOGRAFIA}

Benedetti, G. M., Duval, V. S., \& Campo, A. M. (2016). Propuesta para el análisis de cobertura del arbolado urbano. Caso de estudio: Pigüé, provincia de Buenos Aires. Revista Proyección, 10 (20), 244-258.

Benedetti, G. \& Campo de Ferreras, A. (2007). Arbolado de alineación: el mapa verde de un barrio en la ciudad de Bahía Blanca, Argentina. Papeles De geografía, (45-46), 27-38. Recuperado de: https://revistas.um.es/geografia/article/ view/43081.

Conway, T. M., \& Bourne, K. S. (2013). Una comparación de las características del vecindario relacionadas con la cobertura del dosel, la densidad del tallo y la riqueza de especies en un bosque urbano. Paisaje y planificación urbana, 113, 10-18.doi.org/10.1016/j.landurbplan.2013.01.005.

Demaio, P. H., Medina, M., \& Karlin, U. O. (2002). Árboles nativos del centro de Argentina. Buenos Aires: Ed. L.O.L.A.

Ferro, S. (2004). Introducción a la Arquitectura del Paisaje. Términos y definiciones. Conferencia inédita para la asignatura Introducción a la Arquitectura del Paisaje I. La Habana, Cuba.

Giménez, A. M., \& Moglia, J. G. (2003). Árboles del Chaco Argentino: Guía para el reconocimiento dendrológico (307pp.). Santiago del Estero, República Argentina: Editorial El Liberal S. R. L. ISBN: 987 95852-9-1.

Gómez Piovano, M. J., \& Mesa, N. A. (2015). Análisis de los modos de acceso y los patrones de uso de la población respecto a los espacios verdes urbanos, como base para su planificación. Urbano, 18 (32), 38-49. Recuperado de: http://revistas.ubiobio.cl/index.php/RU/article/view/1995

Grau, A., \& Kortsarz, A. M. (2012). Las especies de árboles de Tucumán. En A., Grau y A. M. Kortsarz. (Eds.), Guía de Arbolado de Tucumán -1ª ed. pp. 109-229. Salta, Argentina: Artes Gráficas Crivelli.

Instituto Nacional de Estadística y Censos de Argentina (INDEC). (2012). Censo Nacional de Población, Hogares y Viviendas 2010. Censo del Bicentenario Resultados definitivos, Serie B № 2. Tomo 1.- 1a ed.-, 1 (378 p). Buenos Aires. Recuperado de: https://www.indec.gov.ar/ftp/cuadros/poblacion/censo2010 tomo1.pdf. 
Magurran, A. E. (1988). Ecological Diversity and Its Measurement (179 pp.). New Jersey: Princeton University Press. ISBN: 0691084912, 9780691084916.

Méndez, E. (2012). Revisión del género Salix (Salicaceae) en la Provincia de Mendoza, Argentina. Revista de la Facultad de Ciencias Agrarias. Universidad Nacional de Cuyo, 44 (2), 157-192. Recuperado de http://www.scielo.org.ar/scielo.php?script=sci_arttext\&pid=S1853866520120002 00013\&lng=es\&tlng=es.

Mills, G., Anjos, M., Brennan, M., Williams, J., McAleavey, C., \& Ningal, T. (2015). La firma verde de las ciudades irlandesas: un examen de los servicios ecosistémicos proporcionados por los árboles utilizando el software i-Tree Canopy. Irish Geography, 48 (2), 62-77. DOI:10.2014/igj.v48i2.625.

Ministerio de Medio Ambiente de la Provincia de Santa Fe (2017). Listado de especies adecuadas para arbolado público en la provincia de Santa Fe. Recuperado de: https://www.santafe.gob.ar/index.php/web/content/download/240740/1269190/fi le/Lista\%20especies_ETFI.pdf.

Moreno, C. E. (2001). Métodos para medir la biodiversidad. M\&T-Manuales y Tesis SEA, vol. 1. Zaragoza, España. ISBN: 84 - 922495 - 2.

Ordenanza N³.385/2000 sobre Nómina de Espacios Verdes Consolidados de la ciudad de Santiago del Estero. Honorable Consejo Deliberante de la Ciudad Capital de Santiago del Estero.

Ordenanza $N^{\circ} 3.823 / 2004$ que trata sobre el "Arbolado Urbano". Honorable Consejo Deliberante de la Ciudad Capital de Santiago del Estero.

Palacio, M. O., \& Roger, E. (2016). Árboles autóctonos de Santiago del Estero: guía para su reconocimiento en el Jardín Botánico Ing. Lucas D. Roic. Santiago del Estero: EDUNSE, Editorial Universitaria.

Roger, E., Generoso M., \& Blanco, R. (2012). Informe de Pasantía FCF-UNSE: Análisis de la vegetación en Plaza San Martin y Plaza Libertad, Santiago del Estero. Inédito.

Roger, E., Generoso, M., Blanco, R., \& Villaverde, A. (2014). Caracterización de la flora leñosa en Plaza Libertad, Santiago del Estero. Quebracho-Revista de Ciencias Forestales, 22 (1-2), 50-56. ISSN: 0328-0543. Recuperado de: https://www.redalyc.org/articulo.oa?id=48133884006. 
Roger, E., Palacio, M., Coria, O., \& Díaz, R. (2016). Notas sobre la flora urbana cultivada en la ciudad de Santiago del Estero, Argentina. Revista Multequina, 25 (1), 1-13. ISSN 1852-7329 on-line. Recuperado de: http://www.scielo.org.ar/ scielo.phpscript=sci_arttext\&pid=S185273292016000100004\&lng=es\&tlng=es.

Roic, L. D., \& Villaverde, A. A. (1999). Árboles y arbustos cultivados en la ciudad de Santiago del Estero, Argentina. Quebracho-Revista de la Facultad de Ciencias Forestales, (7), 79-88.

Villaverde, A., Mazzucco, R., \& Villaverde, G. (2010). Inventario del arbolado urbano público en el barrio Centro de la ciudad de Santiago del Estero. Municipalidad de Santiago del Estero. Santiago del Estero. Argentina. Recuperado de: http://fcf.unse.edu.ar/archivos/quebracho/q7_13.pdf.

Villaverde, A. A., \& Villaverde, G. (2005). Arbolado Urbano Público. En: Fortalecimiento de la Educación Ambiental Regional. Programa "Apoyo a la articulación Universidad - Escuela Media/Polimodal II". Secretaría de Políticas Universitarias del Ministerio de Educación, Ciencia y Tecnología - Facultad de Ciencias Forestales de la UNSE. 47- 64 pp.

Wang, Z. H., Zhao, X., Yang, J., \& Song, J. (2016). Cooling and energy saving potentials of shade trees and urban lawns in a desert city. Applied Energy, 161, 437-444.doi.org/10.1016/j.apenergy.2015.10.047. 